\title{
Segmentation of Lung Cancer using Mark Region Growing and Median Filter
}

\author{
Tarun Thukral \\ M.Tech Scholar \\ Electronics \& Communication Engineering \\ Delhi College of Technology \& Management, \\ Haryana, India \\ Swati Gaur \\ HOD and Thesis Guide \\ Electronics \& Communication Engineering \\ Delhi College of Technology \& Management, \\ Haryana, India
}

\author{
Shradha Verma \\ M.Tech Scholar \\ Electronics \& Communication Engineering \\ Delhi College of Technology \& Management, \\ Haryana, India \\ Nalini Tyagi \\ M.Tech Scholar \\ Electronics \& Communication Engineering \\ Delhi College of Technology \& Management, \\ Haryana, India
}

\begin{abstract}
Lung cancer is the leading cause of cancer-related deaths in western countries. The prognosis for patients with lung cancer depends primarily on the stage of the tumor at the time of clinical diagnosis. This Dissertation presents a method to find and classify the lung tumor by using region growing and Median filter. The Median filter Method are applied for the filtering of lung tumor. Lung cancer is a leading cause of death globally. It is also a major healthcare problem in India. An online search using the words "lung cancer India" yielded the following hits on $20^{\text {th }}$ February 2016. Google gave 43, 80,000 results. In recent years the image processing mechanisms are widely used in several medical areas to improve earlier detection and treatment stages, in which the time factor is very important to discover the disease among the patient as possible as fast. Lung cancer death rates have been the main cause of cancer deaths in the world, early detection and the treatment of lung cancer can greatly improve the survival rate of patient. Historically more men have died than women from lung cancer as a result of higher level of smoking. So the continuous screening test is required to address this problem. The main objective of this work is earlier detection of cancer, often small cancer size to be identified to increase the survival rate. This Dissertation also presents a cost effective approach to classify the normal, malignant and benign tumor using Active shape model, OSF Recurrent neural network with region growing technique. Lung cancer tumor database used for the testing purpose is from the machine learning repository. The highest accuracy of $97.12 \%$ is achieved using the two layer neural network back propagation algorithm.
\end{abstract}

\section{Keywords}

Active shape model, Image processing, OSF, Recurrent neural network, Region growing technique

\section{INTRODUCTION}

Lung cancer is the foremost cause of cancer death for both men and women. More individuals die of lung cancer than of colon, breast, and prostate cancers collective. In 2007, there will be more than 200000 new cases of lung cancer diagnosed in the United States. About 6 of 10 individuals with lung cancer die within 1 year of their diagnosis. These cancers are generally categorized by cell type, such as small cell or non-small cell carcinomas. These categories are used for treatment decisions and determining prognosis (prospect of recovery). In the Past 20 years the incidence and death rates of lung cancer have been taking the top in all distortions, and the occurrence rate is as high as 29,51 per 100 thousand people in our country. All year 20.000 original lung cancer diagnosis happens in Turkey from the public talking of Özdemi (2009) in 2009 National Cancer Week in Turkey. And Özdemir (2009) indicates this illness could be caught in earlier stages $15 \%$ in India and 30\% in USA. For this early detection that reduce the death rate or increase the death ages of the most trustable method for the purpose of initial lung cancer of all determination methods presently available.

But, there are many difficulties in detecting early pathological changes and evaluating oncology parameters in discussing because of the struggle that to date the pathogens of lung cancer is not clear yet. In command to increase the rate of noticing lung nodules, it is using artificial neural networks (ANN) methods to find out the target position in the observed image and to select an adequate pattern image from several reference patterns. CAD systems are planned to procure a second idea, to help not to put any other radiologist. Cancer lung masses often filter the besieging tissue as they have been widened. They separate cancer masses from non-cancer masses in shape, thickness and density (Adhami and Bruce, 1999, pp. 11701177)

\section{METHODOLOGY}

\subsection{RNN (Recurrent Neural Networks):}

The application of feedback enables recurrent networks to acquire state representations. The use of global feedback has the potential of reducing the memory requirement significantly over that of feed-forward neural networks. It is defined as

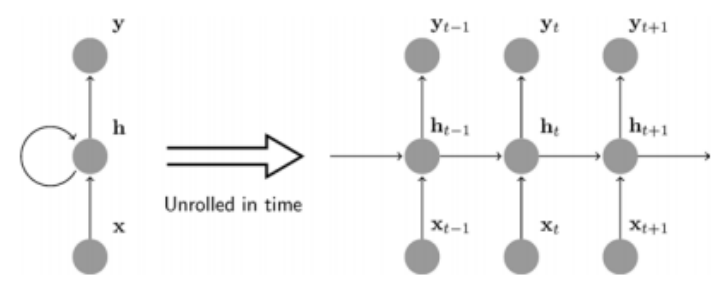

Fig 1: Model of recurrent neural network

Since there are many cases where both past and future inputs have an effect on output for the current input (e.g., in speech recognition), bidirectional recurrent neural networks (BRNNs) have also been designed and used extensively (Fig 2). 


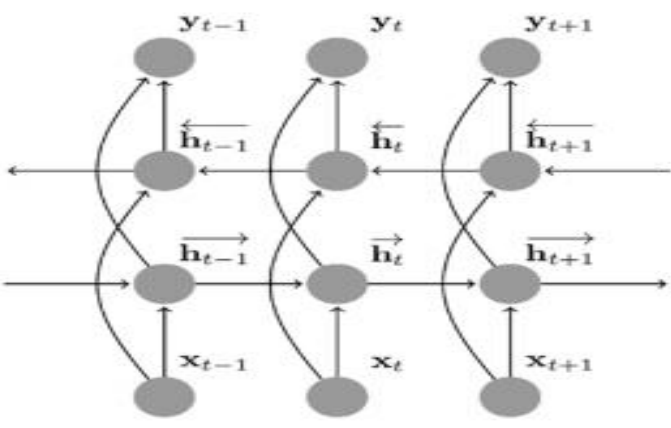

Fig 2: Bidirectional recurrent neural networks

\subsection{Median filter:}

CT scan images are taken. Smoothing of the images includes suppressing the noise and other small fluctuations in the image by using filter Median Filter. Enhancement of the Image is done to improve perception of information in images for human viewers. Lung nodules are smallest growths in the lung that measure between $5 \mathrm{~mm}$ to $25 \mathrm{~mm}$ in size. In abnormal image, it is greater than $25 \mathrm{~mm}$ in size.

\section{LUNG CANCER DETECTION SYSTEM}

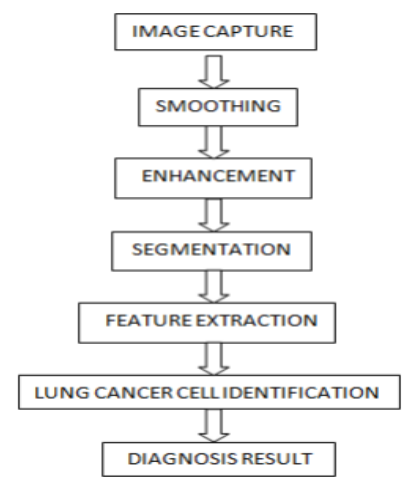

Fig 3: Lung Cancer Detection System

\subsection{Image Acquisition}

Firstly, CT scan image of lung cancer patient is acquired. The lung CT images are having low noise when compared to X-ray and MRI images; hence they are considered for developing the technique.

\subsection{Smoothing}

For smoothing the image, Median Filter is used. Median filtering is a nonlinear method. It is very effective in eliminating noise from images. The median filter works by moving through the image pixel by pixel. It replaces each value with the median value of nearest pixels. This pattern of replacing value of pixels with neighbors is called the "window", which slides, pixel by pixel over the entire image. Then all the pixel values are sorted and arranged in numerical order and then replacing the pixel being considered with the middle (median) pixel value. It suppresses the noise or other small fluctuations in the image. It is equivalent to the suppressions of high frequencies in the frequency domain.

\subsection{Enhancement}

Enhancement technique is used to improve and advance the interpretability or perception of information in images for human viewers.

\subsection{Image Segmentation}

Image segmentation refers to the process of partitioning an image into distinct regions by grouping together neighborhood pixels based on the some predefined similarity criterion. The similarity criterion can be determined using specific properties or features of pixels which represents objects in the image.

\subsection{Feature Extraction}

The entire features obtained provide some information regarding lung nodule. This helps in detecting lung nodule as malignant or nonmalignant.

The area (A) in the object is the just the count of the ones in the image array. For computing area, binary image is used.

$$
\mathrm{A}=\mathrm{N}[1]
$$

\subsection{Region Growing}

The features that are used in this study are consistency and texture features using co-occurrence matrix representation. This method examines neighboring pixels of seed points and determines whether the neighbor pixel should be added to the region or not. The process is iterated in the same manner as common data clustering algorithm. The first step in region growing is to select a set of beginning points which are the points from which tumor has been originated. Beginning points selection is knows as seed point selection. This is based on some user or client criteria for example, pixels in a certain gray scale range, pixels evenly spaced on a grid, etc. The initial region gives us the exact location of these beginning points or seeds.

\subsection{Active Shape Model}

Given a rough starting approximation, an instance of a model can be fit to an image. By choosing a set of shape parameters, $b$ for the model we define the shape of the object in an objectcentered co-ordinate frame. We can create an instance $\mathrm{X}$ of the model in the image frame by defining the position, orientation and scale. An iterative approach to improving the fit of the instance, $\mathrm{X}$, to an image proceeds as follows:

\section{Active Shape Model Algorithm}

1. Examine a region of the image around each point $\mathbf{X}_{i}$ to find the best nearby match for the point $\mathbf{X}_{i}^{\prime}$

2. Update the parameters $\left(X_{t}, Y_{t}, s, \theta, \mathbf{b}\right)$ to best fit the new found points $\mathbf{X}$

3. Apply constraints to the parameters, b, to ensure plausible shapes (eg limit so $\left.\left|b_{i}\right|<3 \sqrt{\lambda_{i}}\right)$.

4. Repeat until convergence.

\section{RESULT ANALYSIS}

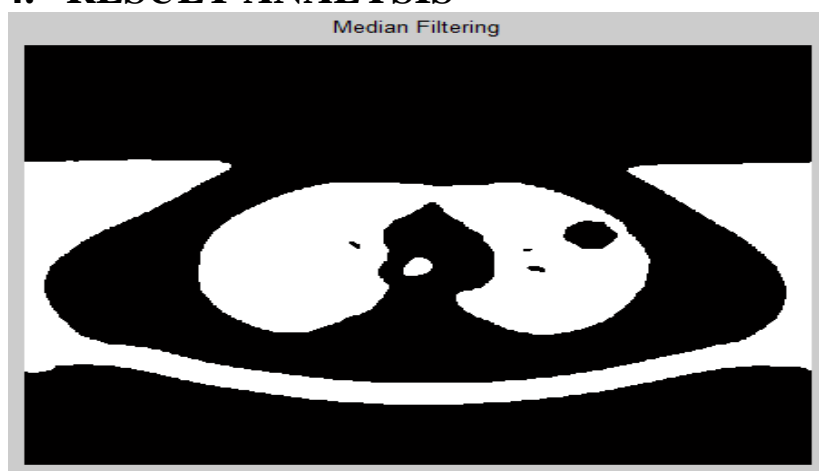

Fig 4: Input Image 
By applying the median filter in Fig 4 there will be suppression of noise and removal of noise, it also preserve the edges of the image which is the main framework in our work. Due to its criteria of storing the edges during smoothing, de-noising method has helped in securing the services of Information. It behaves like a non-linear operator. In according to their Intensity or entropy values the pixels are arranged in a local window by a non-linear operator. At last results show that the value of the pixels replaces the middle value in their specific order and Fig5 is obtained.

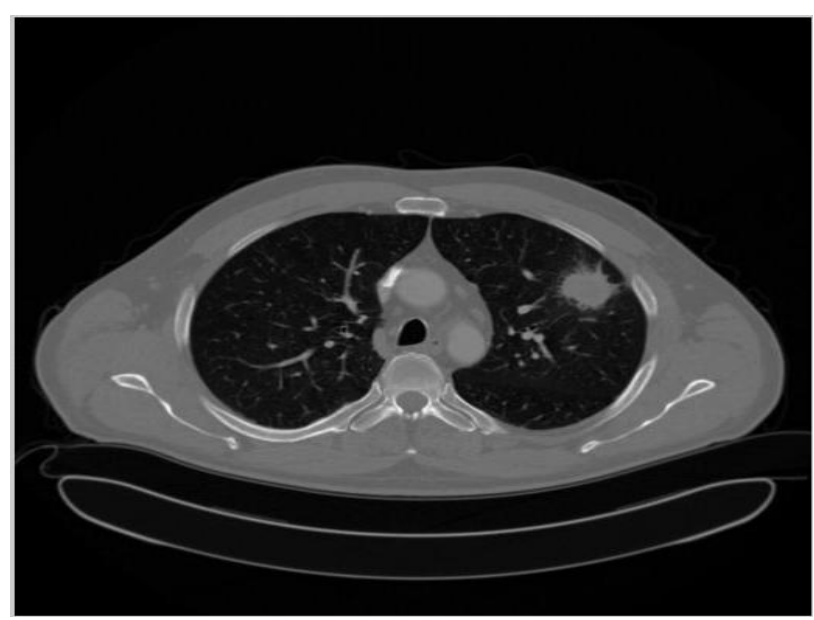

Fig 5: Input image after Median filtering

Well known Image can be seen clearly in gray scale. So Read and convert the Image from RGB to gray scale. It can be done by two methods:

\section{a. Average Method}

$$
\text { scale }=\frac{\mathrm{R}+\mathrm{G}+\mathrm{B}}{3}
$$

b. Weighted method

These three colors have their different wavelengths while forming an image and they contribute in their own fashion. To avoid these turning of black Image it can be overcome by weighted method. We know that wavelength of red color is greater than the remaining two components of the remaining colors. And the Soothing Effect to the eyes is given by the green color. The wavelength of green color is lesser than that of red color. It defines that by decreasing the significance of red color there will be enhancement in the green color .The wavelength of blue color is adjusted between red and green and these gives the new equation.

New gray scale image $=((0.3 * \mathrm{R})+(0.59 * \mathrm{G})+(0.11 * \mathrm{~B}))$.

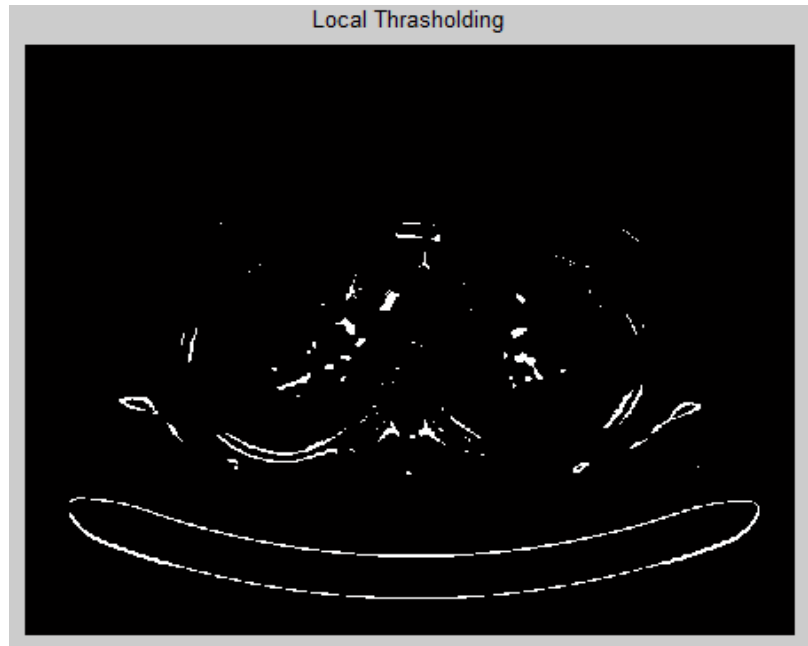

Fig 6: Local thresholding over filtered image

Fig 6 shows the ability of the thresholding and labeling algorithms in determining the actual areas without any loss of representation. To visually observe the accuracy of detection and localization, each labeled area is filled with zeros and projected on the original image. As it clearly appears in the figure, the runlength algorithm was able to detect and localize all possible areas without any losses.

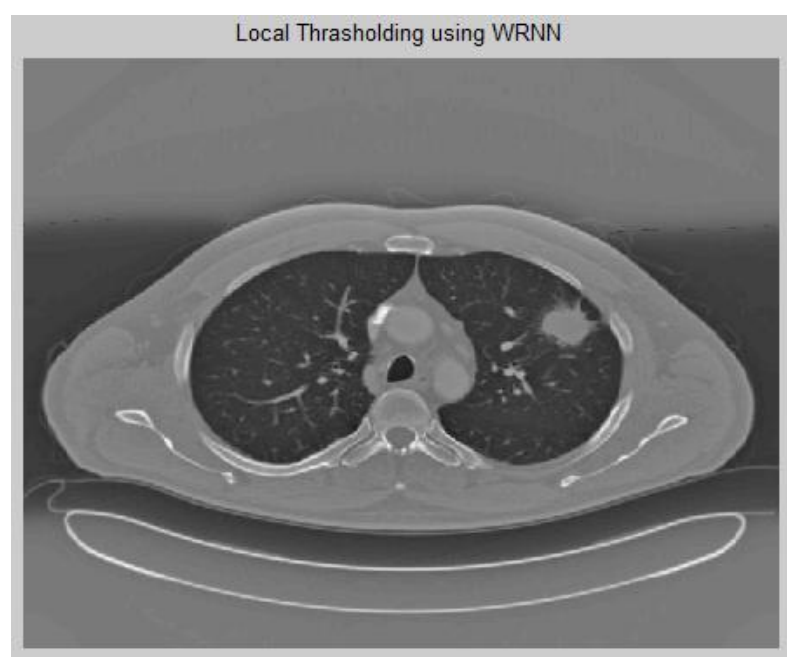

Fig 7: Local thresholding using WRNN

After thresholding, Fig 8 show the enhanced image of original image. The internal morphology of the detected area is a significant indication that enables the radiologist to perform his/her diagnosis using thresholding method. Regions having heterogeneous areas are possible indications of the presence of necrosis, cavitations or calcification. To demonstrate the ability of the proposed system in showing such behavior of the detected tissue, the spatial histogram of the CAD system is used to study the homogeneity of each detected region. 


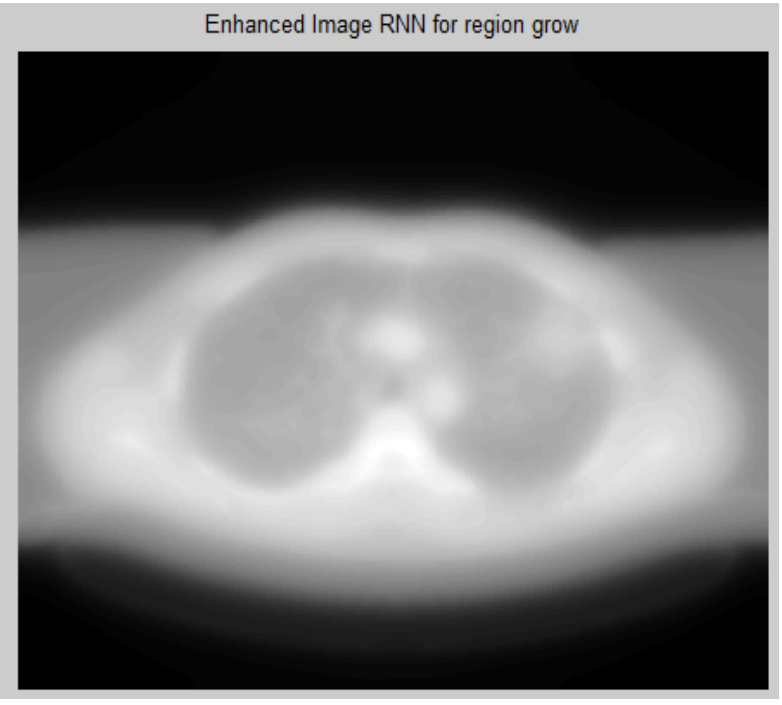

Fig 8: Enhanced image RNN for region

Fig 9 illustrates the different characteristics for the area indicated by a red color in this Figure. To demonstrate the capability of the system to detect small nodules as well as it is capability of detecting large nodules. It shows the margins of the red shade labeled area. The lung segmentation operation to capture lung nodules, however, detecting small nodules is not enough to lead for a real diagnosis; the radiologist needs also the characteristics of each detected nodule.

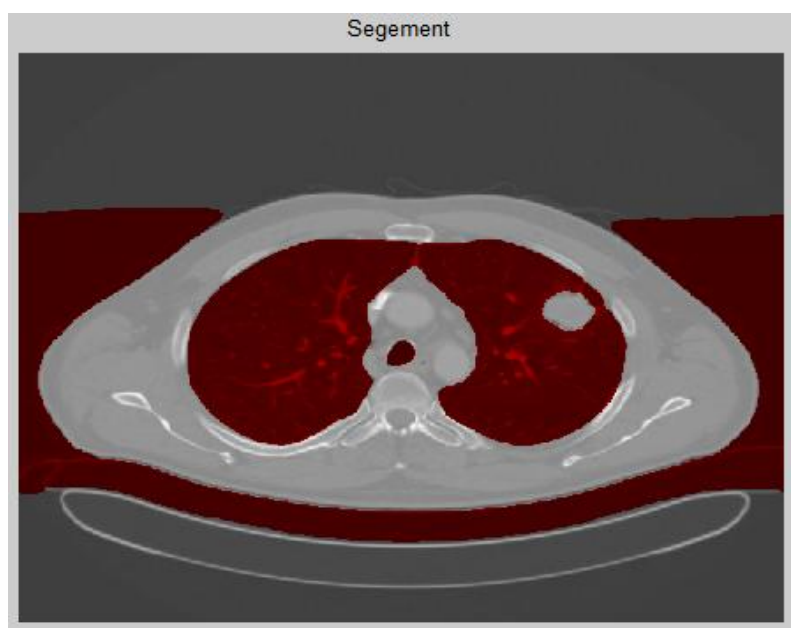

Fig 9: Segmented image after region grow

The goal of this work is to design a local threshold algorithm shown in fig 4.7 that includes shape information to boost the segmentation quality. The algorithm can be divided into two steps: adaptively selecting local threshold based on maximum likelihood, and then removing unnecessary segmented fragments by a supervised classifier. Shape attribute distributions are learned from typical objects in ground truth images. Local threshold for each object in an image to be segmented is chosen to maximize probabilities of these shape attributes according to learned distributions.

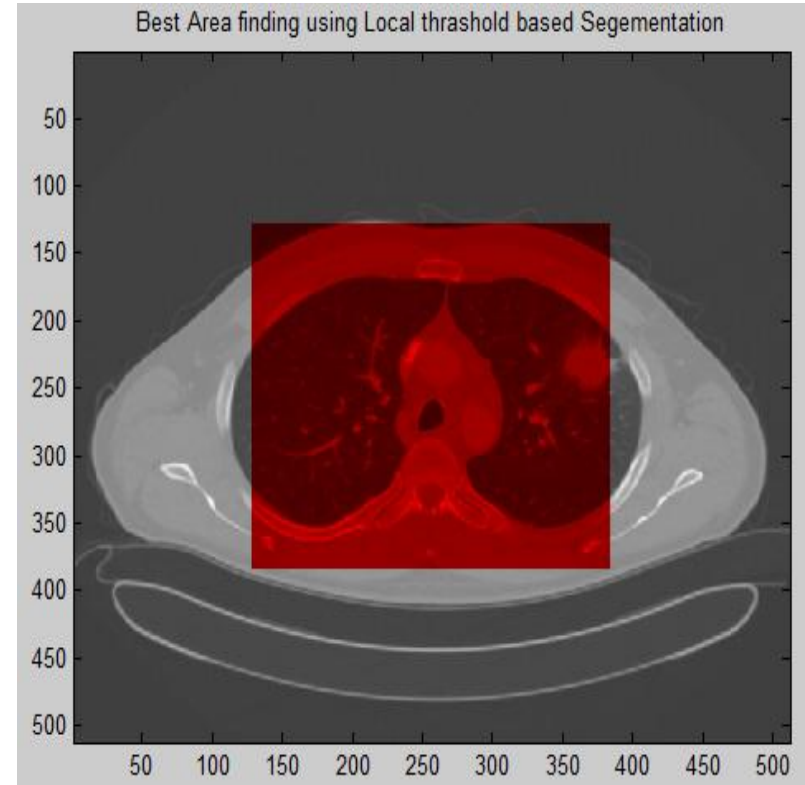

Fig 10: Best area finding using Local threshold based segmentation

The main morphological operations are dilation, erosion, closing, opening and the hit-or-miss transform. Among these apply morphology closing (dilation and erosion) on the image shown in fig 11. It fills the indentation caused by the pulmonary vessels. The effect of dilation is to "grow" or "thicken" objects in a binary image whereas, in erosion, outcome is to "shrink" or "thin" objects. The extent and direction of the thickening and thinning are controlled by the shape and size of the structuring element.

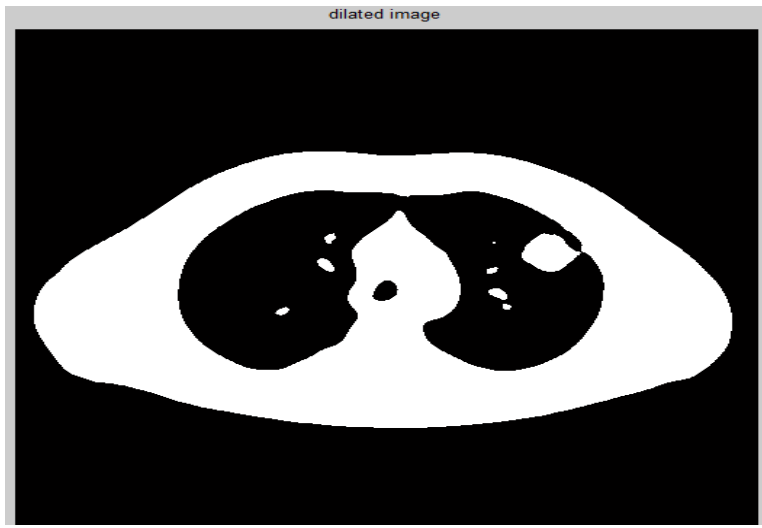

Fig 11: Dilated image

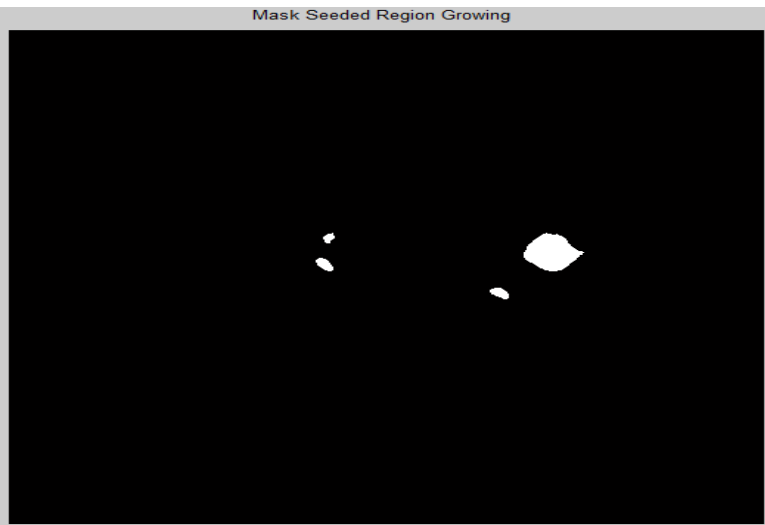

Fig 12: Mask seeded region growing image 
As shown in Fig 12, the Seeded Region Growing is used to extract the size and grey level of certain region of interest on a digital image. The size of the region is calculated as a total number of pixels in the region and given by the following equation. Size $=$ Total of pixels in the region (1). The grey level of the region is calculated as mean value of all pixels in the region and given by the following equation.

\section{Total of grey level for all pixels in the region}

\section{Grey level $=$ \\ Total of pixels in the region}

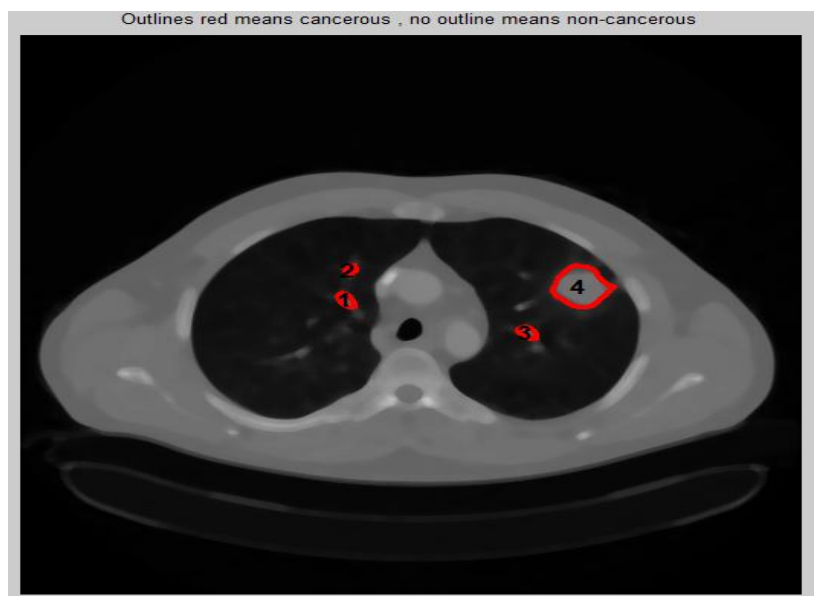

Fig 13: Mask seeded region growing image

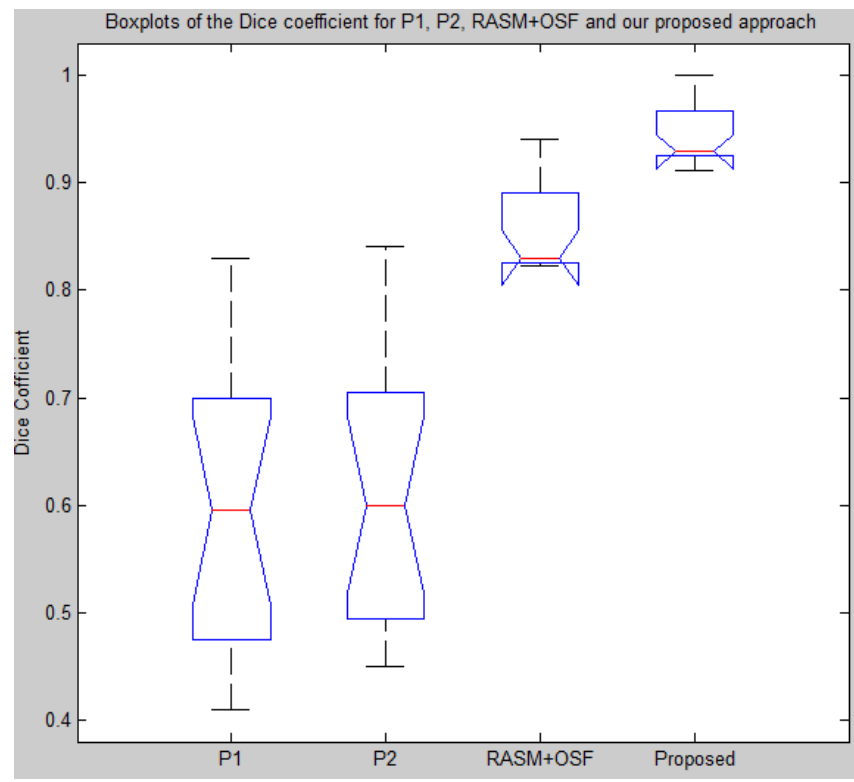

Fig 14: Comparison of Left and Right Lung through P1, P2, Robust active shape modal + OSF and proposed

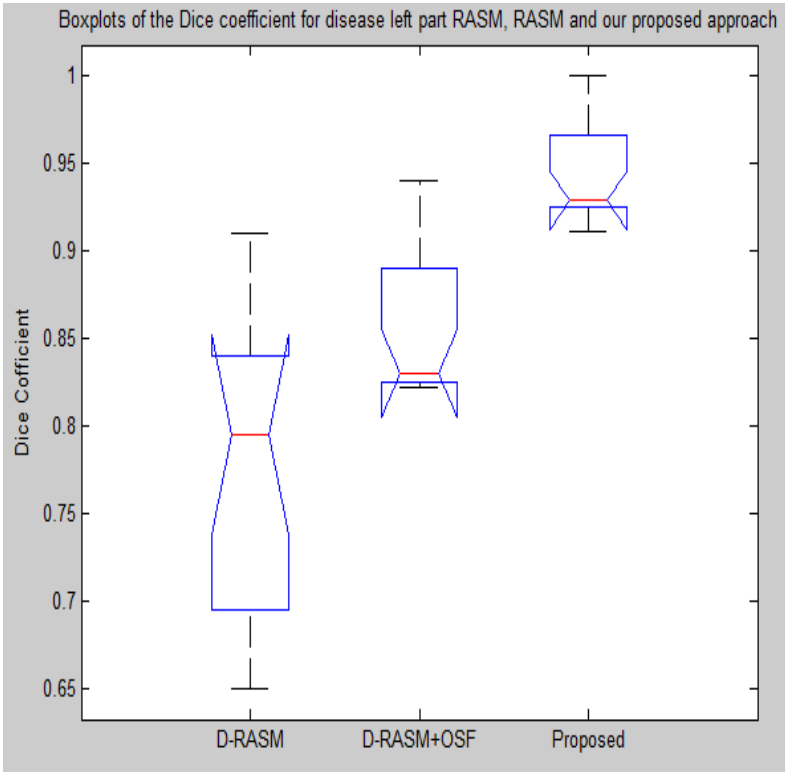

Fig 15: Comparison of Left Lung through DRASM, DRASM+OSF and Proposed

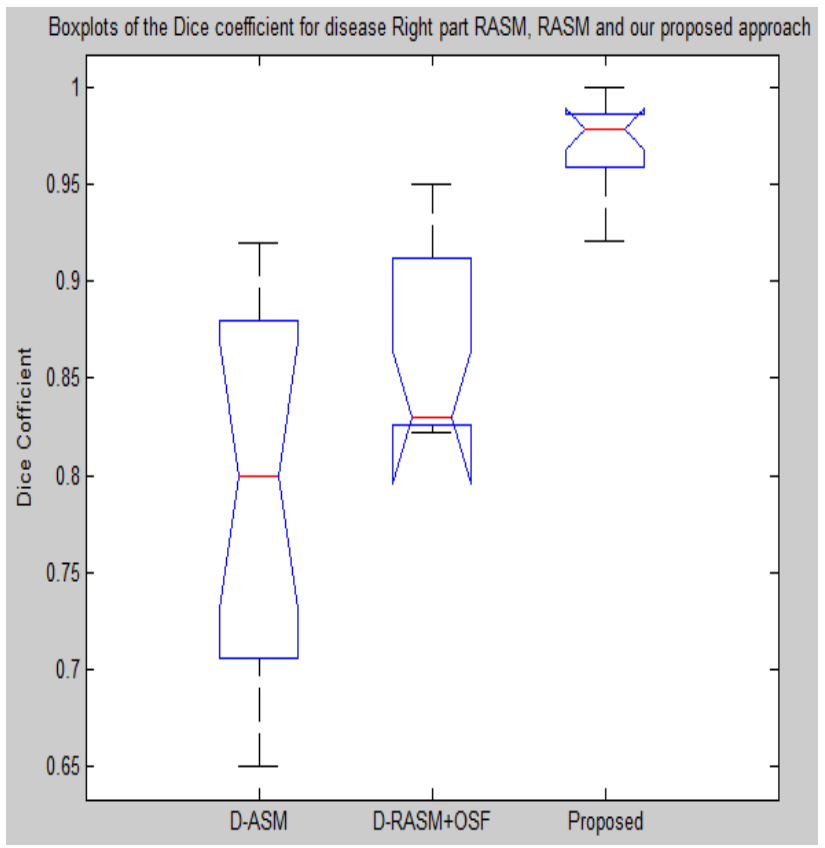

Fig 16: Comparison of Right Lung through D- ASM, DRASM+OSF and Proposed 
Table 1: Results of cancer detected

\begin{tabular}{|c|l|l|l|l|}
\hline Region Number & Area & Perimeter & $\begin{array}{l}\text { Cancer Detected } \\
\text { Centroid }\end{array}$ & Diameter \\
\hline 1 & 105.0 & 36.4 & $214.6,243.4$ & 11.6 \\
\hline 2 & 56.0 & 26.6 & $217.4,216.0$ & 8.4 \\
\hline 3 & 103.0 & 35.6 & $333.4,273.1$ & 11.5 \\
\hline 4 & 1036.0 & 119.3 & $368.3,231.2$ & 36.3 \\
\hline
\end{tabular}

\section{CONCLUSION}

Lung cancer is a major cause of cancer related deaths; it can be detected early by detecting the lung nodules at early stage. The main aim of this paper is to detect the Lung Cancer at an early stage so that treatment can be started early and life of the patient can be saved. Using Median Filter is an effective way of detecting the Lung Cancer at an early stage by enhancing the image by noise reduction. A novel technique was presented in this Dissertation for early detection lung tumor using Median filtering and Region growing. It incorporates Recurrent neural networks in conjunction with advanced image processing procedure as a method which lung cancer diagnosis was performed based on mammogram pictures obtained. The proposed Algorithm showed great success in identifying the region of interest and correctly segmenting all of the input test images. It provides with the proposed Algorithm a very high level of robustness. The results of the proposed method have better accuracy for each Lung cancer stage. The resulting diagnosis showed great promise for being an invaluable and dependable tool for the diagnosis of Lung cancer. Different methods were seamlessly joined together and meshed in a highly technical algorithm which can be considered efficient and very easy to use. Thus, proposed work show a very large area of methods and Techniques can be successfully merged in order to obtain a useful result for human use. This dissertation presented an algorithm to improve the diagnoses of melanoma by the use of image processing and machine vision. The existing system consists of pre-processing, image segmentation, feature extraction and classification. The results showed $97.12 \%$ present accuracy. So we can easily identify cancerous and non-cancerous stage.

\section{REFERENCES}

[1] Jolesz, Ferenc A., Intraoperative Imaging and Image Guided Therapy, Springer: New York Inc, 2014.

[2] Pratibha Sharma, Manoj Diwakar, and Sangam Choudhary, "Application of Edge Detection for BrainTumor Detection," International Journal of Computer Applications, Vol 58, No.16, pp. 21-25, November 2012.

[3] Riries Rulaningtyas, and Khusnul Ain, "Edge Detection For Brain Tumor Pattern Recognition," IEEE International Conference on Instrumentation, Communications, information Technology, and Biomedical Engineering (ICICI-BME),pp. 1 -3, 23-25 Nov. 2009.

[4] ManojDiwakar, Pawan Kumar Patel, and Kunal Gupta, "Cellular Automata Based Edge-Detection For Brain
Tumor," IEEE International Conference on Advances in Computing Communications and Informatics (ICACCI),pp. 53 -59, 22-25 Aug. 2013.

[5] S.M. Ali , LoayKadomAbood , and RababSaadoonAbdoon, "Brain Tumor Extraction in MRI Images using Clustering and Morphological Operations Techniques," International Journal of Geographical Information system Application and Remote Sensing, Vol. 4, No. 1, pp. 1-14, June 2013.

[6] AyshaBava M., and Sifna N. Shajahan, "Segmentation of Brain Tumor in MRI using Multi-structural Element Morphological Edge Detection," International Journal of Engineering Research \& Technology (IJERT), Vol. 3 Issue 3, pp. 313-317, March 2014.

[7] RachanaRana, H.S Badauria, and Annapurna Singh "Study of various methods for brain tumor segmentation from MRI images." International Journal of Emerging Technology and Advanced Engineering. Vol.3, Issue 6, June 2013.

[8] Dong B, Chien A, SHEN Z. Frame based segmentation formedical images. Commun Math Sci 2010;32(4):172439.

[9] Panda M, Patra MR. some clustering algorithms to enhance the performance of the network intrusion detection system. J TheorAppl Inform Technol 2008;4(8):795-801.

[10] Bandhyopadhyay SK, Paul TU. Automatic segmentation of brain tumour from multiple images of brain MRI.Int J ApplInnovatEng Manage (IJAIEM) 2013;2(1):240-8.

[11] Wilson B, Dhas JPM. An experimental analysis of Fuzzy $\mathrm{C}$-means and K-means segmentation algorithm for iron detection in brain SWI using Matlab.Int J ComputAppl 2014;104(15):36-8.

[12] Natarajan P, Krishnan.N, Natasha S, Shraiya N, Bhuvanesh P, "Tumor Detection using threshold operation in MRI Brain Images", International Conference on Computational Intelligence and Computing Research,IEEE,2012.

[13] Manoj K and Sourabh Y, "Brain Tumor Detection and Segmentation Using Histogram Thresholding", International Journal of Engineering and Advanced Technology (IJEAT) ISSN: 2249 -8958, Volume-1, Issue4, April 2012. 
[14] R. B. Dubey, M. Hanmandlu, S. Vasikarla, "Evaluation of Three Methods for MRI Brain Tumor Segmentation", 2011 Eighth International Conference on Information Technology: New Generations, IEEE, 2011.

[15] A. Islam, S. M. S. Reza, and K. M. Iftekharuddin."Multifractal Texture Estimation for Detection and Segmentation of Brain Tumors". In: IEEE Trans Biomed Eng.60 (11) (2013), pp. 3204-3215.

[16] K.Shanthi and M. Kumar. "Skull stripping and automatic segmentation of brain MRI using seed growth and threshold techniques". In: Intelligent and Advanced Systems, 2007. ICIAS 2007.International Conference on. 2007, pp. 422 426.doi:10.1109/ICIAS.2007.

[17] D Kumar and AHalder. "Automatic brain tumor detection and isolation of tumor cells from MRI images". In: Int J ComputAppl 39(16) (2012), pp. 26-30.

[18] J. Kong, J. Wang, Y. Lu, et al. “. A novel approach for segmentation of MRI brain image". In: IEEE MELECON (2006), pp. 525-528.

[19] B. Menze, K. van Leemput, D. Lashkari, et al. "A Generative Model for Brain Tumor Segmentation in MultiModal Images".English. In: Proc MICCAI 2010. 2010, pp. 151 159.isbn: 978-3-642-15744-8. doi:10.1007/978-3-64215745 5_19.url:http://dx.doi.org/10.1007/9783-642-157455_19.

[20] T. Wang, I. Cheng, and A. Basu. "Fully automatic brain tumor segmentation using a normalized Gaussian Bayesian Classifier and 3D Fluid Vector Flow" In: Proc ICIP 2010, pp 2553-2556

[21] C. Garcia and J. Moreno "Kernel based method for segmentation and mod-eling of magnetic resonance images" In: LNCS, 3315. 2004, pp. 636-645.

[22] Gopal, N.N. Karnan, M.,[2010], "Diagnose brain tumor through MRI using image processing clustering algorithms such as Fuzzy C Means along with intelligent optimization techniques ". Computational Intelligence, vol.2 no.1, pp. 1 $-4$
[23] SudiptaRoy , Samir K. Bamdyopadhyay, [2012 ], “ Detection and Quantification of Brain tumoe from MRI of Brain and it's Symmetric Analysis", International Journal of

[24] Information and Communication Technology Research , volume 2 no. 6 , pp.477-483

[25] Marcel Prastawa, Elizabeth Bullitt, Sean Ho, Guido Gerig. "A brain tumor segmentation framework based on outlier detection. Elsevier", Medical Image Analys is, 2004, pp.275 283.

[26] A.Sivaramakrishnan And Dr.M.Karnan "A Novel Based Approach For Extraction Of Brain Tumor In Mri Images Using Soft Computing Techniques", International Journal Of Advanced Research In Computer And Communication Engineering, Vol. 2, Issue 4, April 2013.

[27] .S. Roy And S.K. Bandoyopadhyay, "Detection And Qualification Of Brain Tumor From Mri Of Brain And Symmetric Analysis", International Journal Of Information And Communication Technology Research, Volume 2 No.6, June 2012, Pp584-588.

[28] M. UsmanAkram', Anam Usman2 'Computer Aided System for Brain Tumor Detection and Segmentation' 978-1-61284-941-6/11/\$26.00 @2011 IEEE.

[29] Subhranil Koley and Aurpan Majumder 'Brain MRI Segmentation for Tumor Detection using Cohesion based Self-Merging Algorithm' 9 78-1-61284-486-2/111\$26.00 (C)2011 IEEE

[30] Ahmed KHARRAT Mohamed Ben MESSAOUD, Nacéra BENAMRANE, Mohamed ABID "Detection of Brain Tumor in Medical Images", 2009 International Conference on Signals, Circuits and Systems 978-1-4244-43987/09/\$25.00 @2009 IEEE

[31] S. Parisot, et al., "Graph-based detection, segmentation \& characterization of brain tumors", in Computer Vision and Pattern Recognition (CVPR), 2012 IEEE Conference on, 2012, pp. 988-995 